\title{
Tempo de alimentação e defecação das ninfas de Triatoma melanosoma Martínez, Olmedo \& Carcavallo (Hemiptera, Reduviidae) em diferentes condições de temperatura e umidade
}

\author{
Cleber Galvão ${ }^{1}$ \\ Dayse da Silva Rocha ${ }^{1}$ \\ Vanda Cunha ${ }^{1}$ \\ José Jurberg 1 \\ Rodolfo Carcavallo ${ }^{1}$
}

\begin{abstract}
Feeding and defecation times of nymphs of Triatoma melanosoma Martínez, Olmedo \& Carcavallo (Hemiptera, Reduviidae). The authors observed the feeding and defecation times of the nymphs of T. melanosoma Martínez, Olmedo \& Carcavallo, 1987. Two groups were studied, one maintained under controlled conditions of temperature and humidity, and the other under ambient conditions. There was no significant difference in the number of bloodmeals accomplished by the nymphs of the two groups. Until the fourth instar the nymphs needed less than two bloodmeals to reach the subsequent instar, and only in the fifth instar did they require more than three bloodmeals to reach adulthood. This species demonstrated great avidity to feed; in about $70 \%$ of the observed cases the insects initiated feeding in less than one minute. Full engorgement was also reached quickly; more than $70 \%$ of the bloodmeals lasted for 5 to 15 minutes. In the first three instars more than $70 \%$ of defecation was observed immediately after feeding, demonstrating a good vectorial potential. The differences in the conditions under which the insects were maintained, were insufficient to cause a significant difference in the results observed between the two groups studied.

KEY WORDS. Triatominae, Triatoma melanosoma, feeding and defecation times
\end{abstract}

Os triatomíneos, insetos vetores da doença de Chagas, constituem uma subfamília de hemípteros caracterizados pelo hematofagismo obrigatório em ambos os sexos em todas as fases do desenvolvimento (LENT \& WYGODZINSKY 1979). Atualmente são conhecidas 130 espécies (MEJIA et al. 1999) a grande maioria potencialmente transmissora de Trypanosoma cruzi (Chagas, 1909), o agente etiológico da doença, que é um dos principais problemas de saúde pública na América Latina onde existem 100 milhões de pessoas em áreas de risco e 18 milhões infectadas (WHO 1995) e estima-se que de 15 a 20\% das pessoas infectadas venham a desenvolver clinicamente a doença (WHO 1996).

Os estudos de biologia vem sendo realizados por diversos autores, que determinando características específicas, podem avaliar a competência vetorial, auxiliando

1) Laboratório Nacional e Internacional de Referência em Taxonomia de Triatomineos, Departamento de Entomologia, Instituto Oswaldo Cruz. Avenida Brasil 4365, 21045-900, Rio de Janeiro, Rio de Janeiro, Brasil. E-mail: galvao@gene.dbbm.fiocruz.br 
no planejamento de medidas de controle. Estudos sobre o comportamento alimentar e sobre o tempo de defecação, são indicadores do potencial de transmissão de T. cruzi, possibilitando estimar o grau de importância de cada espécie.

WoOD (1951) foi o primeiro a observar que algumas espécies norte-americanas não defecavam imediatamente após o repasto, demonstrando baixo potencial vetorial. DiAs (1956) ao contrário, observou que as espécies sul-americanas foram mais eficientes neste aspecto. RABINOVICH et al. (1979) concluíram que a agressividade de um triatomíneo para obter a fonte sangüínea, medida através do número de picadas, implica num maior ou menor contato do triatomíneo com o hospedeiro ajudando também a mensurar o potencial vetorial de uma espécie.

Apesar da biologia desses insetos estar sendo estudada desde o início do século, um recente levantamento realizado por CANALE et al. (1999), mostra através de 104 gráficos, dados da biologia das 59 espécies estudadas, demonstrando que até o momento esse número representa menos de $50 \%$ das espécies conhecidas.

Triatoma melanosoma Martínez, Olmedo \& Carcavallo, 1987 é uma espécie afim de Triatoma infestans (Klug, 1834) porém facilmente distinguível por apresentar coloração totalmente negra, é encontrada nos arredores de habitações rurais na província de Misiones, Departamento San Ignacio, no extremo nordeste da Argentina (entre $23^{\circ}$ e $28^{\circ}$ de latitude sul) em região próxima a fronteira do Brasil (CARCAVAllo et al. 1999). Inicialmente descrita como subespécie de T. infestans apresentou estabilidade fenotípica por diversas gerações levando LENT et al. (1994) a elevarem-na ao status de espécie. Recentemente, NOIREAU et al. (1997) descobriram formas silvestres negras de $T$. infestans no chaco Boliviano, entretanto, elas apresentavam marcas claras no conexivo e em outras regiões do corpo. MONTEIRO et al. (1999) não encontraram diferenças genéticas entre $T$. melanosoma e $T$. infestans, porém, não invalidaram a espécie.

A média de duração de ciclo de vida de T. melanosoma foi publicada por CANALE et al. (1999) e a influência da temperatura no seu desenvolvimento foi estudada por GALVÃo et al. (1999), entretanto, ainda não foram publicados trabalhos abordando outros aspectos de sua biologia. Sua afinidade com o T. infestans, o principal vetor da doença de Chagas nos países do Cone Sul, e sua ocorrência próxima a fronteira do Brasil, nos levaram a estudar seus tempos de alimentação e defecação, com o objetivo de avaliar seu potencial vetorial em condições de laboratório. Foram observados: o tempo decorrido entre o oferecimento da fonte e a picada, a duração do repasto, o tempo decorrido entre o término do repasto e a defecação, o local do depósito das dejeções e o número de repastos realizados em cada fase do desenvolvimento em diferentes condições de temperatura e umidade.

\section{MATERIAL E MÉTODOS}

Os insetos utilizados foram provenientes de uma colônia iniciada em 1994 com descendentes de espécimes coletados pelos autores da espécie. No insetário do Laboratório Nacional e Internacional de Referência em Taxonomia de Triatomíneos, do Departamento de Entomologia do Instituto Oswaldo Cruz foram mantidos em temperatura ambiente com alimentação quinzenal em pombos (Columba livia). 
Os ovos foram coletados diariamente da colônia, agrupados em frascos de Borrel ( $9 \mathrm{~cm}$ de altura $\times 3 \mathrm{~cm}$ de diâmetro) de acordo com a data da postura e divididos em dois grupos. $\mathrm{O}$ primeiro foi mantido em estufa a temperatura de $28+/-1^{\circ} \mathrm{C}$ e 80 $+/-5 \%$ de U.R. com fotofase de 12 horas (condições controladas $=$ CC) e o segundo em temperatura ambiente registrada diariamente e umidade variando de 70 a $90 \%$ (condições ambientais $=\mathrm{CA}$ ).

As 30 primeiras ninfas emergidas em cada grupo foram individualizadas em frascos de Borrel numerados, forrados no fundo por papel de filtro contendo tiras do mesmo papel dobradas em "sanfona" para absorver a umidade e proporcionar maior área de deslocamento aos insetos. Os frascos foram fechados com telas de náilon fixadas por elásticos. A alimentação foi oferecida diariamente até a ocorrência do primeiro repasto, passando então a ser semanal. Foi realizada em pombos, que tinham as asas e patas imobilizadas. O tempo de oferecimento da fonte foi de dez minutos e o tempo de sucção cronometrado a partir da picada; após o término do repasto aguardava-se por dez minutos para verificar o tempo decorrido até a defecação. Os resultados foram analisados estatísticamente pelo teste de Mann-Whitney.

\section{RESULTADOS}

\section{Tempo decorrido entre o oferecimento da fonte e a picada}

As tabela I mostra a distribuição de freqüência do tempo decorrido entre o oferecimento da fonte alimentar e a picada. T. melanosoma demonstrou grande avidez para se alimentar; em cerca de $70 \%$ dos casos observados os insetos aceitaram a fonte alimentar em até dois minutos. Este foi o padrão para os dois grupos observados (CC e CA).

Tabela I. Distribuição de freqüências do tempo decorrido entre o oferecimento da fonte e a picada das ninfas de Triatoma melanosoma mantidas em condições controladas e em condições ambientais. (cc) Condições controladas: mantidos em estufa B.O.D. a $28+/-1^{\circ} \mathrm{C}$ e $80+1-5 \%$ de U.R.; (ca) condições ambientais: mantidos em temperatura ambiente (amplitude de 21 a $33^{\circ} \mathrm{C}$ e média de $26^{\circ} \mathrm{C}$ e 70 a $90 \%$ de U.R.).

\begin{tabular}{cccccc}
\hline & \multicolumn{5}{c}{ Número de repastos } \\
\cline { 2 - 6 } Estádios & $0<2$ minutos & $2<4$ minutos & $4<6$ minutos & $6<8$ minutos & $8<10$ minutos \\
\hline $1^{\circ} \mathrm{cc}$ & 28 & 4 & 1 & 0 & 2 \\
$1^{\circ} \mathrm{ca}$ & 28 & 1 & 1 & 0 & 0 \\
$2^{\circ} \mathrm{cc}$ & 33 & 3 & 1 & 0 & 0 \\
$2^{\circ} \mathrm{ca}$ & 37 & 5 & 2 & 0 & 0 \\
$3^{\circ} \mathrm{cc}$ & 40 & 7 & 1 & 0 & 0 \\
$3^{\circ} \mathrm{ca}$ & 38 & 7 & 2 & 0 & 1 \\
$4^{\circ} \mathrm{cc}$ & 35 & 5 & 1 & 0 & 0 \\
$4^{\circ} \mathrm{ca}$ & 44 & 23 & 16 & 6 & 3 \\
$5^{\circ} \mathrm{cc}$ & 43 & 20 & 4 & 1 & 3 \\
$5^{\circ} \mathrm{ca}$ & 82 & & & & 0 \\
\hline
\end{tabular}

\section{Duração do repasto}

No presente trabalho a duração do repasto apresentou padrões muito semelhantes nos dois grupos estudados (CC e CA). No primeiro estádio, em ambos os grupos, $87 \%$ dos repastos duraram de 5 a 15 minutos, no segundo estádio $84 \%$ dos repastos realizados pelo grupo $\mathrm{CC}$ e $86 \%$ dos realizados pelo grupo CA estão nesta 
faixa de duração, já no terceiro estádio os percentuais foram de 87 e $81 \%$ respectivamente. No quarto e quinto estádios houve um aumento na duração dos repastos de ambos os grupos e consequentemente os percentuais obtidos nesta faixa de duração foram reduzidos para 75 e $64 \%$ no quarto estádio e para 66 e $55 \%$ no quinto estádio respectivamente. Em T. melanosoma a marca de 30 minutos foi o limite máximo de duração dos repastos obtidos para algumas ninfas de quinto estádio do grupo CA, no grupo CC o limite foi de 31 minutos (Tab. II).

Tabela II. Distribuição de frequiências de duração do repasto das ninfas de Triatoma melanosoma, mantidas em condiçōes controladas e em condiçōes ambientais. (cc) Condiçōes controladas: mantidos em estufa B.O.D. a $28+/-1^{\circ} \mathrm{C}$ e $80+/-5 \%$ de U.R.; (ca) condições ambientais: mantidos em temperatura ambiente (amplitude de 21 a $33^{\circ} \mathrm{C}$ e média de $26^{\circ} \mathrm{C}$ e 70 a $90 \%$ de U.R.).

\begin{tabular}{lccccccc}
\hline \multirow{8}{*}{ Estádios } & \multicolumn{7}{c}{ Número de repastos } \\
\cline { 2 - 8 } & $0<5$ minutos & $5<10$ minutos & $10<15$ minutos & $15<20$ minutos & $20<25$ minutos & $25<30$ minutos & $30<35$ minutos \\
\hline $1^{\circ} \mathrm{cc}$ & 3 & 23 & 6 & 0 & 1 & 0 & 0 \\
$1^{\circ} \mathrm{ca}$ & 0 & 13 & 13 & 3 & 0 & 1 & - \\
$2^{\circ} \mathrm{cc}$ & 4 & 26 & 7 & 2 & 0 & 0 & 0 \\
$2^{\circ} \mathrm{ca}$ & 5 & 30 & 9 & 0 & 1 & 0 & - \\
$3^{\circ} \mathrm{cc}$ & 0 & 26 & 15 & 6 & 0 & 0 & 0 \\
$3^{\circ} \mathrm{ca}$ & 3 & 23 & 16 & 5 & 1 & 0 & - \\
$4^{\circ} \mathrm{cc}$ & 0 & 18 & 18 & 10 & 2 & 0 & 0 \\
$4^{\circ} \mathrm{ca}$ & 2 & 11 & 21 & 15 & 1 & 0 & - \\
$5^{\circ} \mathrm{cc}$ & 11 & 40 & 29 & 18 & 4 & 1 & - \\
$5^{\circ} \mathrm{ca}$ & 16 & 30 & 30 & 23 & 7 & 3 & - \\
\hline
\end{tabular}

\section{Número de repastos realizados em cada estádio}

$\mathrm{O}$ oferecimento semanal da alimentação permitiu avaliar o número de repastos necessários à ocorrência da ecdise. Não houve diferença significativa entre as médias obtidas em todos os estádios para os dois grupos. No primeiro estádio a média foi de apenas um repasto para ambos os grupos. Do segundo ao quarto estádios as médias dos grupos foram muito próximas e inferiores a dois repastos e somente no quinto estádio foram necessários mais de três repastos para que as ninfas de ambos os grupos atingissem a fase adulta (Tab. III).

Tabela III. Número de repastos realizados em cada estádio de Triatoma melanosoma. (cc) Condições controladas: mantidos em estufa B.O.D. a $28+/-1^{\circ} \mathrm{C}$ e $80+/-5 \%$ de U.R.; (ca) condições ambientais: mantidos em temperatura ambiente (amplitude de 21 a $33^{\circ} \mathrm{C}$ e média de $26^{\circ} \mathrm{C}$ e 70 a $90 \%$ de U.R.); Mann-Whitney, diferenças não significativas, $p<0,01$ ).

\begin{tabular}{ccccccc}
\hline Estádios & Mínimo & máximo & Média & Desvio padråo (S) & Variância (S2) & N \\
\hline $1^{\circ} \mathrm{cc}$ & 1 & 2 & 1,0 & 0,3 & 0,09 & 29 \\
$1^{\circ} \mathrm{ca}$ & 1 & 2 & 1,0 & 0,1 & 0,01 & 30 \\
$2^{\circ} \mathrm{cc}$ & 1 & 2 & 1,3 & 0,4 & 0,16 & 28 \\
$2^{\circ} \mathrm{ca}$ & 1 & 3 & 1,5 & 0,5 & 0,25 & 29 \\
$3^{\circ} \mathrm{cc}$ & 1 & 3 & 1,7 & 0,5 & 0,25 & 28 \\
$3^{\circ} \mathrm{ca}$ & 1 & 2 & 1,5 & 0,5 & 0,25 & 28 \\
$4^{\circ} \mathrm{cc}$ & 1 & 3 & 1,7 & 0,5 & 0,25 & 29 \\
$4^{\circ} \mathrm{ca}$ & 1 & 6 & 1,7 & 1,0 & 1,00 & 25 \\
$5^{\circ} \mathrm{cc}$ & 2 & 7 & 3,7 & 1,3 & 1,69 & 27 \\
$5^{\circ} \mathrm{ca}$ & 1 & 6 & 3,6 & 1,5 & 2,25 & \\
\hline
\end{tabular}




\section{Padrões de defecação}

A figura 1 demonstra que nos dois grupos estudados o percentual de defecações realizadas imediatamente após o repasto, sobre a fonte alimentar, foi alto superando os $70 \%$ nos três primeiros estádios, decrescendo no quarto e quinto estádios. Essa tendência de inversão nos percentuais de defecação, pode ser atribuída ao aumento do número de repastos onde o ingurgitamento completo não ocorreu. Esses resultados indicam que sob este aspecto T. melanosoma apresenta um grande potencial vetorial.
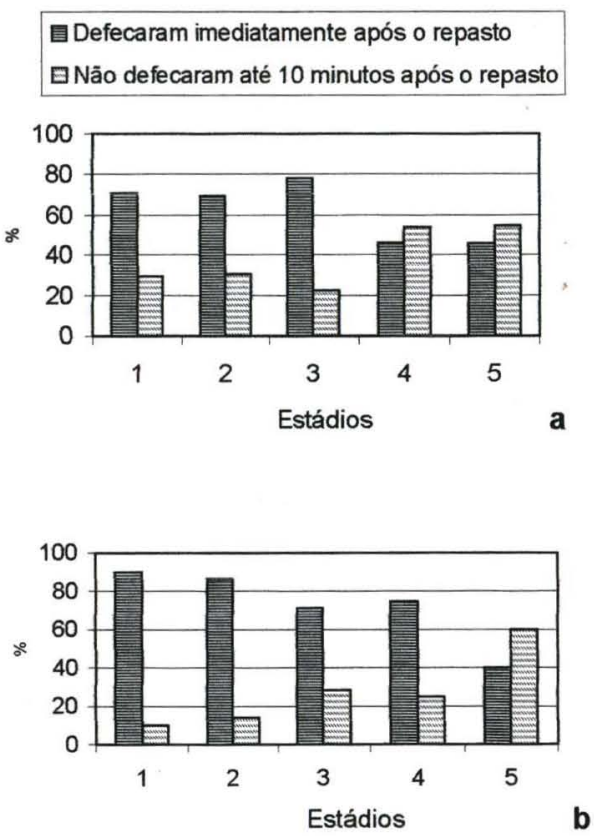

Fig. 1. Tempos de defecação das ninfas de Triatoma melanosoma. (a) mantidas em condições controladas; (b) mantidas em temperatura e umidade ambientais.

\section{DISCUSSÃO}

O registro do tempo decorrido entre o oferecimento fonte e a picada permite avaliar a voracidade na procura pelo alimento. GonÇALVES et al. (1988), observando o tempo de aceitação da fonte em Triatoma vitticeps (Stål, 1959) obtiveram tempos muito superiores aos obtidos no presente trabalho, pois em apenas $12 \%$ dos casos do primeiro ao quinto estádios o ato de picar foi imediato. Se comparado com Triatoma nitida Usinger, 1939 estudado por GALVÃo et al. (1995) T. melanosoma também teve uma melhor performance, já que naquela espécie em $60 \%$ dos repastos observados, o intervalo entre o oferecimento e a picada foi inferior a 4,5 minutos BRAGA \& LiMA (1999) observando as ninfas de Triatoma rubrofasciata (De Geer, 1773) obtiveram médias variando de 7 a 11 minutos para a aceitação da fonte. A 
voracidade de T. melanosoma superou até mesmo a de uma espécie de Rhodnius, gênero conhecido por sua extrema agressividade. A média obtida por RocHA et al. (1994) para Rhodnius pictipes Stål, 1872 foi de 53\% dos repastos iniciando-se em até dois minutos. DiOTAIUTI et al. (1995) observaram o tempo gasto entre a liberação das ninfas de terceiro estádio de $T$. infestans e o início do repasto em grupos alimentados na parte da manhã e ao final da tarde, a média obtida foi de nove minutos para os primeiro grupo e de 18 minutos para o segundo. CANALS et al. (1999) comparando alguns parâmetros de comportamento alimentar de $T$. infestans e Mepraia spinolai Porter, 1939, verificaram que esta espécie apresentou um comportamento agressivo, iniciando rapidamente a alimentação que durou em média nove minutos, tempo muito inferior a média obtida para $T$. infestans (19 minutos).

A rápida detecção de possíveis fontes alimentares, bem como rapidez na realização do repasto são extremamente importantes para o sucesso do desenvolvimento dos triatomíneos, já que espécies que conseguem se alimentar rapidamente, causando menos incômodo aos seus hospedeiros levam uma grande vantagem sobre aquelas que não o fazem. O nível de incômodo ao hospedeiro causado pelo processo de alimentação, não tem maior importância em espécies silvestres encontradas em pequenas densidades (FoRATTINI 1980), entretanto, em domicílios onde geralmente são encontradas em grandes densidades (DIAS \& ZELEDÓN 1955; RABINOVICH et al. 1979) podem determinar uma diminuição na quantidade de sangue ingerido, afetando a dinâmica populacional e o potencial vetorial das espécies. Segundo SCHOFIELD et al. (1986) um aumento na densidade causa uma maior percepção, pelo hospedeiro, das picadas sofridas diminuindo a quantidade de sangue sugado devido as seguidas interrupções no repasto. Essas afirmações foram corroboradas por PEREIRA et al. (1995) que demonstraram experimentalmente que $T$. infestans é mais eficiente que $P$. megistus na obtenção de sangue em camundongos não anestesiados.

A realização do repasto com conseqüente defecação é o meio mais comum de exposição do hospedeiro a T. cruzi. Quanto mais tempo um vetor demora se alimentando sobre um hospedeiro infectado, maior será a possibilidade de sua infecção por tripanosomas. Diante desse quadro, os estudos sobre padrões de alimentação e defecação, adquirem grande importância para ampliar os conhecimentos sobre a transmissão de T. cruzi e apontar as espécies de maior potencial vetorial. WOOD (1951) foi o primeiro a realizar experimentos sobre tais hábitos. DIAS (1956) estudou seis espécies sul-americanas e demonstrou que Rhodnius prolixus Stål, 1859 foi a espécie que apresentou o menor tempo de sucção, 14 minutos, e o maior percentual de defecações durante o repasto, verificou ainda, que $T$. vitticeps foi a espécie que sugou durante o maior período e que não defecou durante, ou logo após o repasto. O tempo de sucção e a quantidade de sangue ingerido foi observado por PIPPIN (1970) para T. sanguisuga Usinger, 1944, T. gerstaeckeri (Stål, 1859) e R. prolixus, mostrando que R. prolixus têm um potencial de transmissão de T. cruzi maior que as outras duas espécies. ZELEDón et al. (1977) compararam os tempos de defecação de $R$. prolixus, $T$. infestans e $T$. dimidiata (Latreille, 1811), concluindo que R. prolixus foi superior as outras espécies no número de defecações realizadas. ZÁrATE et al. (1984) demonstraram que só 13\% das defecações de T. barberi Usinger, 1939 ocorreram durante o repasto. Segundo

Revta bras. Zool. 18 (Supl. 1): 233 - 242, 2001 
BRASIlEIRo \& PERONDINI (1974) nas ninfas de quarto e quinto estádios de $T$. brasiliensis Neiva, 1911 até os 30 minutos de sucção o aumento da quantidade ingerida é aproximadamente linear, a partir daí, o aumento é menos significativo até os 60 minutos de sucção, em média, a repleção já é atingida a partir dos 30 minutos.

A determinação da freqüência alimentar ideal e da freqüência crítica é importante tanto do ponto de vista epidemiológico quanto para otimizar as criações em laboratório (CABELLO et al. 1988). RocHA et al. (1994) observaram que as ninfas de $R$. pictipes necessitaram em média de 1,6 repastos, quando alimentados em pombos, para alcançarem a fase imaginal, essa média foi de dois repastos quando os insetos foram alimentados em camundongos por RocHA et al. (1997). O número de repastos realizados tem implicâncias epidemiológicas, já que quanto mais contatos o vetor e o hospedeiro mantiverem, maior será a probabilidade de sua infecção ou transmissão de T. cruzi. Por outro lado quanto menos vezes o vetor se expuser saindo a procura de um hospedeiro, maiores serão suas chances de sobrevivência. Muitas espécies podem atingir até o quarto estádio com apenas um repasto por estádio, como foi discutido por HACK (1955). GoODCHILD (1955) observou em $T$. infestans a necessidade de mais de um repasto por estádio a partir do terceiro; para dar continuidade ao ciclo. Os espécimes de T. brasiliensis e Triatoma pseudomaculata Corrêa \& Espínola, 1964 observados por SoARES et al. (2000) apresentaram uma inversão no número habitual de repastos por estádio, já que as médias obtidas para o primeiro estádio (três repastos) foram superiores as obtidas para o quinto estádio (dois repastos), em ambas as espécies.

PERLOWAGORA-SZUMLEWICZ (1953) conseguiu abreviar o período de desenvolvimento de $T$. infestans alimentando-a a cada quatro dias. FREITAS et al. (1967) demonstraram que com alimentação semanal o período de desenvolvimento de Rhodnius neglectus Lent, 1954 foi mais curto quando comparado com a alimentação quinzenal; a mesma relação foi obtida por CABELLO et al. (1988) para adultos de Rhodnius neivai Lent, 1953.

O tempo que o inseto leva para defecar é crucial para a transmissão de $T$. cruzi e varia de acordo com as espécies (PIESMAN \& SHERLOCK 1983; ZÁRATE et al. 1984); é um dos principais indicadores da capacidade vetorial, embora existam outros fatores como: densidade, adaptação ao domicílio e antropofilia, que auxiliam na determinação dessa capacidade (LENT \& WYGODZINSKY 1979; PIESMAN \& SHERLOCK 1983). Isto tem motivado diversos autores a estudarem os tempos de defecação dos principais vetores da doença de Chagas. A maior eficácia da transmissão se dá nas espécies que defecam durante, ou logo após o repasto, e já foi demonstrada entre outros por DIAS (1956), ZELEDÓN et al. (1977), OZETE (1981), ZÁrATE et al. (1984), KIRK \& SCHOFIELD (1987), GonÇALVES et al. (1988), COSTA \& Jurberg (1990), Rocha et al. (1994), GAlvão et al. (1995) e CANALS et al. (1999). DIAS (1956) observou que apenas 10\% das ninfas de T. infestans defecaram durante, ou logo após o repasto, enquanto que nos adultos este percentual foi de cerca de $50 \%$, percentuais iguais e superiores a este foram observados por SOARES et al. (2000) para as ninfas de quarto e quinto estádios e adultos de T. brasiliensis e T. pseudomaculata. CROCCO \& CATALÁ (1996) estudando ninfas de quinto estádio 
e adultos de T. sordida (Stål, 1959) observaram defecações durante o repasto e freqüência máxima nos primeiros dez minutos após a alimentação, o que não foi observado para T. pseudomaculata por GoNÇAL VES et al. (1997) já que na defecação dessa espécie a demora na maioria dos casos foi superior a 10 minutos demonstrando baixa competência vetorial. A comparação dos tempos de defecação entre as espécies estudadas deve levar em conta as diferenças nas metodologias utilizadas e a grande variabilidade individual intraespecífica, mesmo assim é possível, baseado nos resultados obtidos traçar perfis de espécies mais, ou menos competentes na transmissão de $T$. cruzi.

AGRADECIMENTOS. Ao CNPq, FAPERJ e convênio FNS/Fiocruz n 123/97 pelo auxílio financeiro. Ao Prof. Octavio Augusto França Presgrave pelas análises estatísticas. A dois consultores anônimos pelas críticas e sugestões.

\section{REFERÊNCIAS BIBLIOGRÁFICAS}

Br^GA, M.V. \& M.M. Lima. 1999. Feeding and defecatiom patterns of nymphs of Triatoma rubrofasciata (De Geer, 1773) (Hemiptera: Reduviidae), and its potential role as vector for Trypanosoma cruzi. Mem. Inst. Oswaldo Cruz 94: 127-129.

Brasileiro, V.L.F. \& A.L.P. PerondinI. 1974. Biologia do Triatoma brasiliensis (Hemiptera, Reduviidae, Triatominae) I. Tempo de sucção e repleção de ninfas de $4^{\circ}$ e $5^{\circ}$ estádio. Revta bras. Ent. 18: 43-50.

Cabello, D.R.; E. Lizano \& A. Valderrama. 1988. Efecto de la frecuencia alimentaria sobre algunos parametros poblacionales de Rhodnius neivai. Mem. Inst. Oswaldo Cruz 83: 441-446.

Canals, M.; R. Solís; C. TApia; M. Ehrenfeld \& P.E. CAtTA. 1999. Comparison of Triatoma infestans Klug, 1834 and Mepraia spinolai Porter, 1939, vectors of Chagas disease in Chile. Mem. Inst. Oswaldo Cruz 94: 687-692.

Canale, D.; J. Jurberg; R.U. Carcavallo; C. Galvão; I. Galíndez-Girón; C.A. Mena-Segura; D.S. Rocha \& A. Martínez. 1999. Bionomics of some species. Bionomia de algumas espécies, p. 839-890. In: R.U. CARCAVAllo; I. Gai.índeZ-Girón; J. Jurberg \& H. Lent (Eds). Atlas of Chagas' disease vectors in the America. Atlas dos vetores da doença de Chagas na Américas. Rio de Janeiro, Ed. Fiocruz, Vol. 3,1217p.

Carcavallo, R.U.; S.I. Curto de Casas; I.A. Sherlock; I. Galindez-Girón; J. Jurberg; C. Galvão; C.A. MEnA-SEgura \& F. Notrenu. 1999. Geographical distribution and alti-latitudinal dispersion. Distribuição geográfica e dispersão altilatitudinal, p. 747-792. In: R.U. CARCAVAlLO; I. Galindez-Girón; J. Jurberg \& H. Lent (Eds). Atlas of Chagas' disease vectors in the America. Atlas dos vetores da doença de Chagas na Américas. Rio de Janeiro, Ed. Fiocruz, Vol. 3, 1217p.

Costa, J.M. \& J. Jurberg. 1990. Estudos bionômicos de Cavernicola lenti Barrett \& Arias, 1985 (Hemiptera, Reduviidae, Triatominae). Mem. Inst. Oswaldo Cruz 85: 356-366.

CROCCO, L.B. \& S.S. CATALÁ. 1996. Feeding and defaecation patterns in Triatoma sordida. Mem. Inst. Oswaldo Cruz 91: 409-413.

DiAS, E. 1956. Observações sôbre o tempo de evolução de algumas espécies de Triatomíneos em laboratório. Mem. Inst. Oswaldo Cruz 54: 115-124.

DiAS, E. \& R. ZELEDÓN. 1955. Infestação domiciliária em grau extremo por Triatoma infestans. Mem. Inst. Oswaldo Cruz 53: 473-486.

Diotaiutı, L.; C.M. PEnido; H.H. PIRES \& J.C.P. DiAs. 1995. Dinâmica da alimentação e dejeção do Triatoma sordida. Rev. Soc. Brasil. Med. Trop. 28: 195-198.

ForatTINI, O.P. 1980. Biologia, origem e distribuição da domiciliação de triatomíneos no Brasil. Rev. Saúde Públ. 14: 265-299.

Revta bras. Zool. 18 (Supl. 1): 233 - 242, 2001 
FrLitAS, M.G.; J.O. Costa \& H.M.A CostA. 1967. Alguns aspectos da biologia do Rhodnius neglectus Lent, 1954 (Hemiptera, Triatominae) em condições de laboratório. I Evolução. Arq. Esc. Vet., Rio de Janeiro, 19: 81-87.

Galvão, C.; J. JurberG; V. Cunha \& R.P. Mello. 1995. Biologia do Triatoma nitida Usinger, 1939 em laboratório (Hemiptera, Reduviidae). Mem. Inst. Oswaldo Cruz 90: 657-663.

Galvão, C.; D.S. Rocha; V. Cunha; O.A.F. Presgrave; J. Jurberg \& R.U. Carcavallo. 1999. Influência da temperatura no ciclo de vida de Triatoma melanosoma Martínez, Olmedo \& Carcavallo, 1987 (Hemiptera, Reduviidae). Mem. Inst. Oswaldo Cruz 94: 851-854.

Goodchild, A.J.P. 1955. Some observations on growth and egg production of the blood sucking Reduviids Rhodnius prolixus and Triatoma infestans. Proc. Royal Ent. Soc. London 30: 137-144.

Gonçalves, T.C.M.; V. Cunha; E. DE. Oliveira \& J. Jurberg. 1997. Alguns aspectos da biologia de Triatoma pseudomaculata Corrêa \& Espinola, 1964, em condições de laboratório (Hemiptera: Reduviidae: Triatominae). Mem. Inst. Oswaldo Cruz 92: 275-280.

Gonçalves, T.C.M; V.M.N. VictóRIO; J. JuRBerg \& V. CUnHA. 1988. Biologia do Triatoma vitticeps (Stål, 1859) em condições de laboratório (Hemiptera: Reduviidae: Triatominae). I. Ciclo evolutivo. Mem. Inst. Oswaldo Cruz 83: 519-523.

HACK, W.H. 1955. Estudios sobre biologia del Triatoma infestans (Klug, 1834) (Hem., Reduviidae). Ann. Inst. Med. Reg. 4: 125-147.

KIRK, M.L. \& C.J. SCHOFIELD. 1987. Density-dependent timing of defaecation by Rhodnius prolixus, and its implications for the transmission of Trypanosoma cruzi. Trans. R. Soc. Trop. Med. Hyg. 81: 348-349.

Lent, H; J. Jurberg; C. Galvão; R.U. Carcavallo. 1994. Triatoma melanosoma, novo status para Triatoma infestans melanosoma Martínez, Olmedo \& Carcavallo, 1987. Mem. Inst. Oswaldo Cruz 89: 353-358.

Lent, H. \& P. Wygodzinsky. 1979. Revision of the Triatominae (Hemiptera, Reduviidae) and their significance as vectors of Chagas' disease. Bull. Amer. Mus. Nat. Hist. 163: 123-520.

MeJiA, J.M.; C. Galvão \& J. JuRBERG. 1999. Rhodnius colombiensis sp. n. da Colômbia, com quadros comparativos entre estruturas fálicas do gênero Rhodnius Stål, 1859 (Hemiptera, Reduviidae, Triatominae). Entomol. Vect. 6: 601-617.

Monteiro, F.; R. Perez; F. Panzera; J.P. Dujardin; C. Galvão; D.S. Rocha; F. Noireau; C. SChofield \& C.B. BEARD. 1999. Mitochondrial DNA variation of Triatoma infestans populations and its implication on the specific status of T. melanosoma. Mem. Inst. Oswaldo Cruz 94: 229-238.

Noireau, F.; R. Flores; T. Gutierrez \& J.P. Dujardin. 1997. Detection of sylvatic dark morphs of Triatoma infestans in the Bolivian Chaco. Mem. Inst. Oswaldo Cruz 92: 583-584.

OzETE, H.J. 1981. Observaciones sobre la biología de Triatoma flavida Neiva, 1911 en Cuba. Rev. Cub. Med. Trop. 33: 42-50.

Pereira, M.H.; C.M. Penido; M.S. Martins \& L. Diotaiuti. 1995. Triatoma infestans is more efficient than Panstrongylus megistus in obttaining blood meals on non anaesthetized mice. Mem. Inst. Oswaldo Cruz 90: 765-767.

Perlowagora-Szumlewicz, A. 1953. Ciclo evolutivo do Triatoma infestans em condições de laboratório. Rev. Bras. Malariol. D. Trop. 5: 35-47.

Piesman, J. \& I.A. SherLock. 1983. Factors controlling the volume produced by triatominae vectors of Chagas' disease. Acta Trop. 40: 351-358.

PIPIN, W.F. 1970. The biology and vector capability of Triatoma sanguisuga texana Usinger and Triatoma gerstaeckeri (Stål) compared with Rhodnius prolixus (Stål) (Hemiptera: Triatominae). Jour. Med. Ent. 7: 30-45.

RABINOVICH, J.E.; J.A. LEAL \& D.F. PINERO. 1979. Domiciliary bitingfrequency and blood ingestion of the Chagas'disease vector Rhodnius prolixus Stål (Hemiptera: Reduviidae), in Venezuela. Trans. Royal Soc. Trop. Med. Hyg. 73: 272-283.

Rocha, D.S.; A.H. Fonseca; F.A. Costa; J. Jurberg \& C. Galvăo. 1997. Desenvolvimento de Rhodnius pictipes Stål, 1872 alimentado através de membrana de silicone e em camundongos 
(Hemiptera, Reduviidae, Triatominae). Mem. Inst. Oswaldo Cruz 92: 553-558.

Rocha, D.S.; C. GALVÃo \& J. JuRBERG. 1994. Biologia do Rhodnius pictipes Stål, 1872 em condições de laboratório (Hemiptera, Reduviidae, Triatominae). Mem. Inst. Oswaldo Cruz 89: 265-270.

SChofield, C.J.; N.G. Williams \& T.F. MARShall. 1986. Density dependent perception of Triatominae bug bites. Ann. Trop. Med. Parasit. 80: 351-358.

Soares, R.P.P.; L.G. Evangelista; L.S. Laranja \& L. Diotaiuti. 2000. Population dynamics and feeding behavior of Triatoma brasiliensis and Triatoma pseudomaculata, main vectors of Chagas disease in northeastern Brazil. Mem. Inst. Oswaldo Cruz 95: 151-155.

Wно (World Health Organization). 1995. Chagas' disease: important advances in elimination of transmission in four countries in Latin America. Geneva, WHO (Press office, feature 183). . 1996. Climate change and human health. Geneva, WHO, 298 pp.

WooD, S.F. 1951. Importance of feeding and defecation times of insect vectors in transmission of Chagas'disease. Jour. Econ. Ent. 44: 52-54.

Zárate, L.G.; G.M. López; M.C. Ozuna; G.G. Santiago \& R.J. Zárate. 1984. The biology and behaviour of Triatoma barberi (Hemiptera: Reduviidae) in Mexico. IV. Feeding and defecation patterns. Jour. Med. Entomol. 21: 548-560.

ZELEDÓN, R.; R. Alvarado \& L.F. JiRÓN. 1977. Observations on the feeding and defecation patterns of three triatominae species (Hemiptera: Reduviidae). Acta Trop. 34: 65-77.

Recebido em 14.IX.2000; aceito em 26.VI.2001. 